NISSUNA UMANA INVESTIGAZIONE SI PUO DIMANDARE VERA SCIENZIA S'ESSA NON PASSA PER LE MATEMATICHE DIMOSTRAZIONI LEONARDO DA VINCI

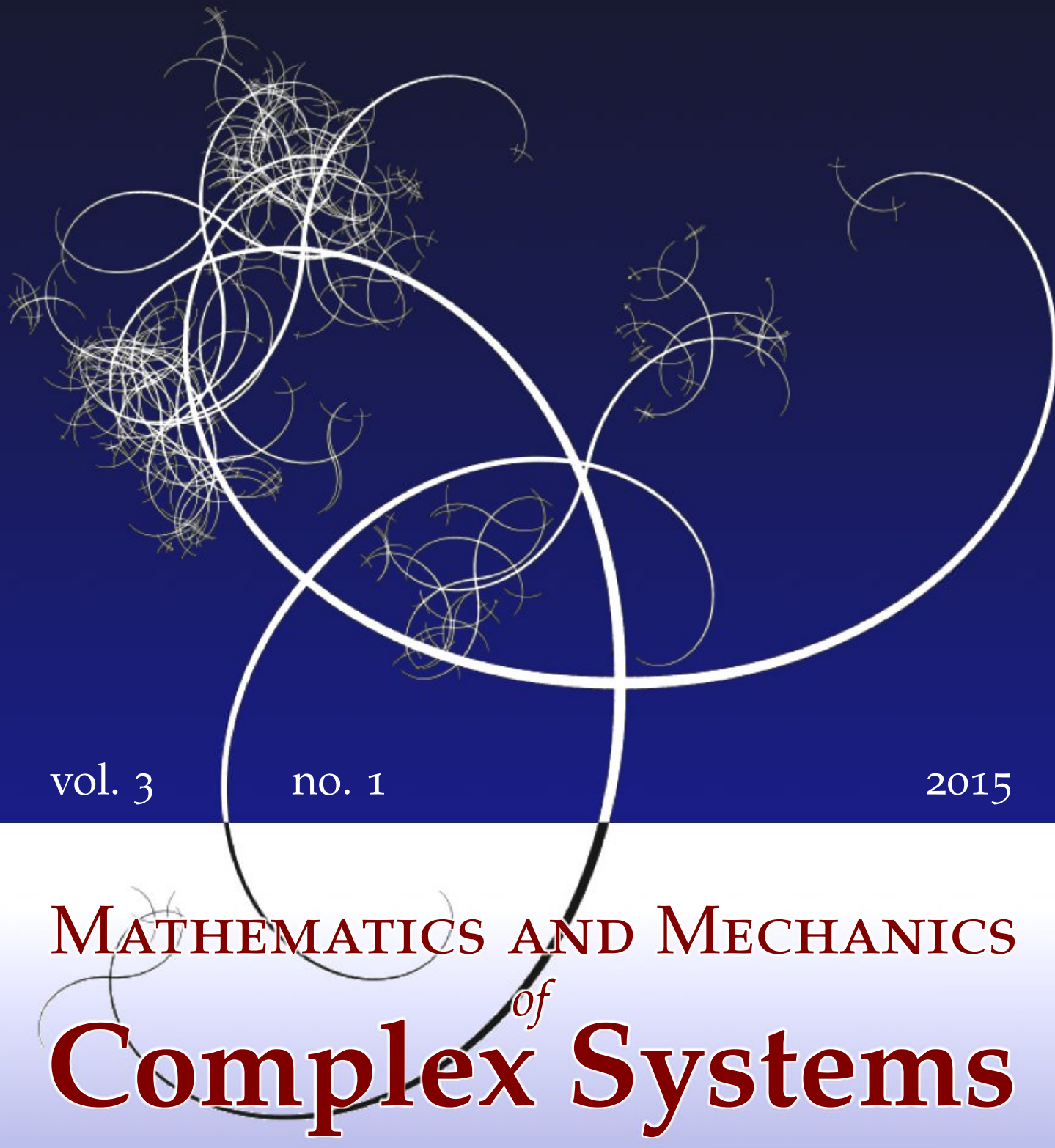

Lihua Shen, Martin Ostoja-Starzewski And Emilio Porcu RESPONSES OF FIRST-ORDER DYNAMICAL SYSTEMS TO MATÉRN, CAUCHY, AND DAGUM EXCITATIONS 


\title{
RESPONSES OF FIRST-ORDER DYNAMICAL SYSTEMS TO MATÉRN, CAUCHY, AND DAGUM EXCITATIONS
}

\author{
Lihua Shen, Martin Ostoja-Starzewski AND Emilio PorCU
}

\begin{abstract}
The responses of dynamical systems under random forcings is a well-understood area of research. The main tool in this area, as it has evolved over a century, falls under the heading of stochastic differential equations. Most works in the literature are related to random forcings with a known parametric spectral density. This paper considers a new framework: the Cauchy and Dagum covariance functions indexing the random forcings do not have a closed form for the associated spectral density, while allowing decoupling of the fractal dimension and Hurst effect. On the basis of a first-order stochastic differential equation, we calculate the transient second-order characteristics of the response under these two covariances and make comparisons to responses under white, Ornstein-Uhlenbeck, and Matérn noises.
\end{abstract}

\section{Introduction}

A vast amount of research in mathematics, physics, and mechanics has, since the time of Einstein, Langevin, and Smoluchowski, been motivated by the responses of dynamical systems under random forcings. The main tool used in this area, as it has evolved over a century of investigations, falls under the heading of stochastic differential equations. While it seems that linear stochastic dynamical systems (that is, those governed by linear differential equations) form a very well-established body of knowledge, the subject of such systems driven by wide-sense stationary (WSS) random noises with no Fourier transforms has not been explored. The point is that, when dealing with a WSS process, all studies tacitly assume a spectral density exists. However, this is not that case with WSS processes - and, generally, WSS random fields in $\mathbb{R}^{3}$ — with either Cauchy [Gneiting and Schlather 2004] or Dagum [Porcu et al. 2007] covariance functions. An additional intriguing fact about the Cauchy and Dagum functions is that they can model fractal as well as Hurst effects. Roughly speaking, the former is a roughness measure of a profile

\section{Communicated by Antonio Carcaterra.}

MSC2010: primary 34F05, 60H10; secondary 28A80, 62H10.

Keywords: random dynamical system, stochastic ordinary differential equation, fractal, Hurst effect. 
(that is, a realization on the real line) or surface of $\mathbb{R}^{n}$, whilst the latter reflects possible long-memory dependence in a time series or a random field.

The celebrated works [Matheron 1965; Stein 1999; Christakos 2000] (along with the references therein) illustrate how several properties of random fields enjoying Cauchy or Dagum covariance can be studied through their correlation functions. In particular, the local and global behavior is sketched in the next sections.

While fractals are quite well known as "those enchanting, self-similar things" [CFA], the Hurst effect, being less well known, warrants a few words here. The effect is modeled by an exponent $H$, which, in the context of a time series, is a measure of long-term memory. While $0<H<0.5$ indicates a time series with negative autocorrelation (for example, a decrease between values will likely be followed by an increase), $0.5<H<1$ indicates a time series with positive autocorrelation (an increase between values followed by another increase). The case $H=0.5$ indicates a true random walk, where there is no preference for a decrease or increase following any particular value.

We consider the transient response of a linear, time-invariant system obeying the equation

$$
\begin{aligned}
c X^{\prime}+k X & =c(\beta+\gamma t) U(t) F(t), \\
X(0) & =0,
\end{aligned}
$$

to a wide-sense stationary random excitation $F(t)$ having either a white noise, Ornstein-Uhlenbeck (OU), Matérn, Cauchy, or Dagum covariance function. In (1) $c, k, \beta$, and $\gamma$ are deterministic constants, while $U(t)$ is the Heaviside function:

$$
U(t):= \begin{cases}1 & \text { if } t \geqslant 0, \\ 0 & \text { if } t<0 .\end{cases}
$$

Letting $a=k / c$ and $Y(t)=(\beta+\gamma t) U(t) F(t)$ we have

$$
X^{\prime}+a X=Y(t) .
$$

It is easy to see that the specific solution $X(t)$ of the above ordinary differential equation can be expressed as

$$
X(t)=\int_{0}^{t} h_{a}(t-\tau) Y(\tau) \mathrm{d} \tau,
$$

where $h_{a}(t)=e^{-a t} U(t), a>0$, is the elementary solution of this ordinary differential equation. We assume that $\mathbb{E}[F(t)]=0$, which in turn implies $\mathbb{E}[X(t)]=0$. For simplicity, we shall make use of the special case $\beta=1, \gamma=0$ in most parts of the paper, without loss of generality.

Our objective in this study is to determine the second-order characteristics of $X(t)$ (and make relative comparisons), assuming that $F(t)$ is a Gaussian random process with either a white noise, OU, Matérn [Matérn 1986], generalized Cauchy 
[Gneiting and Schlather 2004], or Dagum [Porcu et al. 2007] covariance function. The intriguing thing about generalized Cauchy and Dagum covariances is that they are natural decouplers of fractal dimension and Hurst effects, in the sense that the associated Gaussian random process is not self-similar. This, in turn, has considerable advantages from the statistical viewpoint, since the parameters indexing fractal dimension and the Hurst effect can be estimated separately. For many facts on these classes of covariance functions and their properties in terms of fractal dimension and the Hurst effect, the reader is referred to the survey in [Porcu and Stein 2012].

Of course, white noise and Matérn have no Hurst effects (and white noise is not even a fractal). We include them in our study because the former is the most well-known random noise, while the latter is proposed as superior for multiscale modeling.

The plan of the paper is as follows: In Section 2 we review the basic facts on the covariance functions of Cauchy and Dagum types, including their fractal dimensions and Hurst effects. In Sections 3 and 4, respectively, we compute the variance and correlation structure of responses $X(\cdot)$ for five different random forcings $F(\cdot)$.

\section{Background}

2.1. Covariance functions, fractal dimension, and the Hurst effect. This section is largely expository and reports the basic facts needed for a better understanding of the subsequent sections. As stated through Section 1, the process $F(\cdot)$ in (1) is a zero-mean second-order stationary Gaussian random process defined on the real line, so that its distribution is completely specified by its associated covariance function $C(\cdot, \cdot): \mathbb{R} \times \mathbb{R} \rightarrow \mathbb{R}$, defined as

$$
C\left(t_{1}, t_{2}\right):=\operatorname{Cov}\left(F\left(t_{1}\right), F\left(t_{2}\right)\right), \quad t_{1}, t_{2} \in \mathbb{R}
$$

As a consequence of the assumption of second-order stationarity, there exists a mapping $C_{F}: \mathbb{R}_{+} \cup\{0\} \rightarrow \mathbb{R}$ such that

$$
C\left(t_{1}, t_{2}\right)=C_{F}\left(\left|t_{1}-t_{2}\right|\right)
$$

Such a framework allows us to identify some important properties of the random processes we want to study.

The local properties of a time series or a surface of $\mathbb{R}^{n}$ are related to the fractal dimension, $D$, which is a roughness measure with range $[n, n+1)$. Higher values indicate rougher surfaces. Long memory in a time series or spatial data is associated with power law correlations, and is often referred to as the Hurst effect. 
Long-memory dependence is characterized by the $H$ parameter [Mateu et al. 2007]. Let us see how these properties relate to those of the associated correlation function.

As far as the local behavior is concerned, in the weakly stationary (read: secondorder stationary) case, if, for some $\alpha \in(0,1)$,

$$
\lim _{r \rightarrow 0}\left(C_{F}(0)-C_{F}(r)\right) r^{-\alpha}=K, \quad 0<K<\infty, \quad r>0,
$$

then, with probability one, the random process $F(\cdot)$ satisfies

$$
D=\operatorname{dim}(\operatorname{Gr} F)=\min \left(\frac{1}{\alpha / 2}, 1-\alpha / 2\right),
$$

where, as before, $C_{F}$ denotes the covariance function of $F$. Here, Gr $F$ denotes $\operatorname{graph}(F)=\{(t, F(t)), t \in[-1,1]\} \subset \mathbb{R}^{2}$. Thus, the estimate of $\alpha$ determines that of the fractal dimension $D$. Equation (5) refers to the issue of scaling laws, which describe the way in which rather elementary measurements vary with the size of the measurement unit, and we refer to [Hall and Wood 1993] for a detailed analysis of the relation between the fractal index $\alpha$ and the fractal dimension $D$, as well as to the work in [Adler 1981] on Gaussian index- $\beta$ random fields, with $\beta=\alpha / 2$ in this case.

On the other hand, if, for some $\beta \in(0,1)$,

$$
\lim _{r \rightarrow \infty} C_{F}(r) r^{-1+\beta}=1,
$$

then the process is said to have long memory, with Hurst coefficient $H=\beta / 2$. For $H \in(1 / 2,1)$ or $H \in(0,1 / 2)$ the correlation is said to be, respectively, persistent or antipersistent. In the spectral domain, under the conditions stated in the tauberian and abelian theorems, the interpretation of parameters $\alpha$ and $\beta$ is given in the opposite fashion, so that the same properties can be studied with respect to the Fourier transform of the covariance function, called the spectral density. Basically, the parameter $\alpha$ is associated with the velocity of decay of the spectral density, while the parameter $\beta$ is associated with the local behavior of the spectral density in the neighborhood of zero frequencies.

2.2. Parametric classes for the process $\boldsymbol{F}(\cdot)$. Throughout the paper we shall examine how the response $X(\cdot)$ is affected by random excitation and in what ways it is sensitive to specific classes of covariance functions that allow (or don't allow) it to index fractal dimensions and the Hurst effect. We shall make use of the following functions:

(i) White noise. In this case $F$ is a Gaussian white noise, and its covariance is written as

$$
C_{\mathrm{WN}}(r):=\delta(r), \quad r \geqslant 0,
$$

with $\delta$ denoting the Dirac delta function. 
(ii) Ornstein-Uhlenbeck. In this case $F$ is an Ornstein-Uhlenbeck process (denoted $F=\mathrm{OU}$ ), and its covariance function is of the negative exponential type. It is written as follows:

$$
C_{\mathrm{OU}}(r ; v):=\frac{v}{2} \mathrm{e}^{-v r}, \quad r \geqslant 0,
$$

where $v$ is a positive scaling parameter and where we parametrized $C_{\mathrm{OU}}$ in such a way that

$$
\lim _{\nu \rightarrow \infty} C_{\mathrm{OU}}(\cdot ; v)=C_{\mathrm{WN}}(\cdot) .
$$

(iii) Matérn [1986]. A Gaussian process $F$ has a Matérn covariance if

$$
C_{\mathcal{M}}(r ; v):=r^{v} \mathscr{K}_{\nu}(r), \quad r \geqslant 0,
$$

where $v$ is a parameter that determines the smoothness at the origin of $C_{\mathcal{M}}$, and thus the mean square differentiability of $F$. Here $\mathscr{K}_{v}$ is a modified Bessel function of order $v$. Special cases of interest are

- $C_{\mathcal{M}}(r ; 1 / 2)=\mathrm{e}^{-r}$,

- $C_{\mathcal{M}}(r ; 3 / 2)=(1+r) C_{\mathcal{M}}(r ; 1 / 2)$, and

- $C_{\mathcal{M}}(r ; 5 / 2)=\left(1+r+3 r^{2} / 2\right) C_{\mathcal{M}}(r ; 1 / 2)$.

(iv) Generalized Cauchy [Gneiting and Schlather 2004]. In this case,

$$
C_{\mathscr{C}}(r ; \theta, \eta):=\left(1+r^{\theta}\right)^{-\eta / \theta},
$$

where $\eta>0$ and $0<\theta \leqslant 2$ are necessary and sufficient conditions for positive definiteness. Special cases of this class will also be of interest. In particular, $C_{\mathscr{C}}(\cdot, 2, \gamma)$ is the characteristic function of the symmetric Bessel distribution, $C_{\mathscr{C}}(\cdot, \alpha, \alpha)$ is the characteristic function of the Linnik distribution, and $C_{\mathscr{C}}(\cdot, 1, \gamma)$ is the symmetric generalized Linnik characteristic function [RuizMedina et al. 2011].

(v) Dagum [Porcu et al. 2007]. In this case,

$$
C_{\mathscr{D}}(r ; \delta, \epsilon):=1-\left(1+r^{-\delta}\right)^{-\epsilon / \delta},
$$

where $0<\epsilon<\delta$ and $0<\delta \leqslant 2$ are sufficient conditions for positive definiteness.

Some comments are in order. The Cauchy and Dagum models have been chosen for the present study because they allow us to treat independently the fractal dimension $D$ and the Hurst effect $H$ of their associated random process $F$. In particular, it can be shown [Gneiting and Schlather 2004] that the Cauchy covariance in (10) behaves like (5) for $\theta \in(0,2]$ and like (6) for $\eta \in(0,1)$, whilst the Dagum model in (11) behaves like (5) for $\epsilon \in(0,2]$ and like (6) for $\delta \in(0,1)$, although some caution is needed because we work under the restriction $\epsilon \leq \delta$. Anyway, another sufficient condition is $\delta \in(0,2]$ and $\epsilon \in(0,1]$ [Mateu et al. 2007]. Another useful 
sufficient condition in $\mathbb{R}^{3}$ is $\theta<(7-\epsilon) /(1+5 \epsilon)$ and $\epsilon<7$. Since these two models decouple $(D, H)$, the associated random process will not be self similar in the sense of Mandelbrot, and in general we shall have $D+H \neq 2$ (recall that we are working with profiles here).

The Matérn covariance in (9) indexes the fractal dimension $D$ but has light tails, so that it is not useful for indexing phenomena with long-range dependence.

\section{The variance of $X(\cdot)$}

Equation (4) implicitly shows that the variance of the response $X$ is evolutionary in time (that is, nonstationary). Assuming $\beta=1$ and $\gamma=0$, we have

$$
\mathbb{E}\left[X^{2}(t)\right]=\int_{0}^{t} \int_{0}^{t} C_{F}\left(t_{1}-t_{2}\right) h_{a}\left(t-t_{1}\right) h_{a}\left(t-t_{2}\right) \mathrm{d} t_{1} \mathrm{~d} t_{2},
$$

with $h_{a}$ defined through (4) and where $C_{F}$ is the covariance function associated with $F$, which can be one of the five choices proposed in previous section.

Let us now show how these variances vary from one case to another.

(i) White noise. If $C_{F}=C_{\mathrm{WN}}$, the calculation of the variance in (4) is straightforward. In fact, if $F=\mathrm{WN}$ we have $\mathbb{E}[F]=0$ and $S(\omega)=S_{0}<\infty$, where $S$ denotes the Fourier transform of $C_{\mathrm{WN}}$ and $S_{0}$ is an arbitrary constant. Without loss of generality, we let $S_{0}=1 /(2 \pi)$ so that

$$
\int_{-\infty}^{+\infty} C_{\mathrm{WN}}(t) \mathrm{d} t=1
$$

We thus have (see [Elishakoff 1983, Equation (9.104), p. 348])

$$
\begin{aligned}
\mathbb{E}\left[X^{2}(t)\right]=2 \pi S_{0} e^{-2 a t}\left\{\frac{\beta^{2}}{2 a}\left(e^{2 a t}-1\right)+2 \beta \gamma\right. & {\left[\frac{e^{2 a t}}{4 a^{2}}(2 a t-1)+\frac{1}{4 a^{2}}\right] } \\
& \left.+\frac{\gamma^{2}}{4 a^{3}}\left(e^{2 a t}\left(2 a^{2} t^{2}-2 a t+1\right)-1\right)\right\} .
\end{aligned}
$$

(ii) Ornstein-Uhlenbeck. In the case $F=O U$, the variance of $X(t)$ is

$$
\mathbb{E}\left[X^{2}(t)\right]=\int_{0}^{t} \int_{0}^{t} C_{\mathrm{OU}}\left(t_{1}-t_{2}\right) h_{a}\left(t-t_{1}\right) h_{a}\left(t-t_{2}\right) \mathrm{d} t_{1} \mathrm{~d} t_{2} .
$$

Direct computation yields the following special cases:

$$
\begin{aligned}
& \text { if } a=v=1, \quad \mathbb{E}\left[X^{2}(t)\right]=\frac{1}{4}-\frac{1}{4} \mathrm{e}^{-2 t}\left(1+\mathrm{e}^{2 t}\right), \\
& \text { if } a=1, v \neq 1, \quad \mathbb{E}\left[X^{2}(t)\right]=\frac{1}{-1+v} \mathrm{e}^{-t} v \sinh (t), \\
& \text { if } a \neq 1, a \neq v, \quad \mathbb{E}\left[X^{2}(t)\right]=\frac{v}{2\left(a^{3}-a v^{2}\right)}\left[a-v+(a+v) \mathrm{e}^{-2 a t}-2 a e^{-(a+v) t}\right] .
\end{aligned}
$$




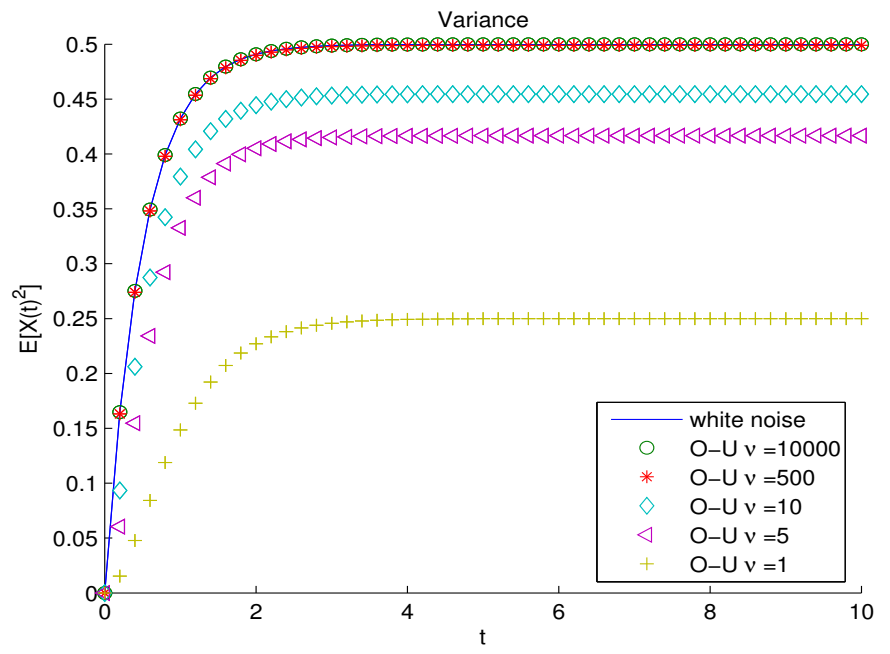

Figure 1. The variances of the response $X(t)$ under white noise and Ornstein-Uhlenbeck (OU) forcings. The white noise curve overlaps with the OU process curves for $v=10,000$ and $v=500$.

Figure 1 depicts $\mathbb{E}\left[X^{2}(t)\right]$ with different values of $v$ and compares it with the variance from the white noise. Here we let $a=1$. Note that the variance caused by the OU process goes to the variance caused by the white noise when $v$ is large enough for $F(t)$ approaching white noise.

(iii) Matérn. If $C_{F}=C_{\mathcal{M}}$, the calculation of the variance is not available in a closed form due to the presence of the modified Bessel function $\mathscr{K}$ in (9). Thus, we choose the case $C_{F}=C_{M}(\cdot ; 3 / 2)$ so that, for $a \neq 1$,

$$
\begin{aligned}
\mathbb{E}\left[X^{2}(t)\right]= & \int_{0}^{t} \int_{0}^{t} C_{\mathcal{M}}\left(t_{1}-t_{2} ; 3 / 2\right) h_{a}\left(t-t_{1}\right) h_{a}\left(t-t_{2}\right) \mathrm{d} t_{1} \mathrm{~d} t_{2} \\
= & \int_{0}^{t}\left(\int_{0}^{t_{2}}+\int_{t_{2}}^{t}\right) C_{\mathcal{M}}\left(t_{1}-t_{2} ; 3 / 2\right) \mathrm{e}^{-a\left(t-t_{1}\right)} \mathrm{e}^{-a\left(t-t_{2}\right)} \mathrm{d} t_{1} \mathrm{~d} t_{2} \\
= & \frac{a}{\left(a^{2}-1\right)^{2}}\left[2-3 a+a^{3}\right. \\
& \left.\quad+\mathrm{e}^{-2 t}\left(-2-3 a+a^{3}\right)+\mathrm{e}^{-t-t / a}\left(6 a-2 a^{3}+2 t-2 a^{2} t\right)\right] .
\end{aligned}
$$

For $a=1$, a straightforward computation gives

$$
\mathbb{E}\left[X^{2}(t)\right]=\frac{1}{4}\left(3+\mathrm{e}^{-2 t}\left(-2 t^{2}-6 t-3\right)\right) .
$$

The same calculations can be performed using the Fourier transform of the Matern function and then invoking basic Fourier calculus; the details are omitted for the sake of simplicity. 
(iv) Generalized Cauchy. If $C_{F}(\cdot)=C_{\mathscr{C}}(\cdot, \theta, \eta)$ then

$$
\mathbb{E}\left[X^{2}(t)\right]=\int_{0}^{t} \int_{0}^{t} C_{\mathscr{C}}\left(t_{1}-t_{2} ; \theta, \eta\right) h_{a}\left(t-t_{1}\right) h_{a}\left(t-t_{2}\right) \mathrm{d} t_{1} \mathrm{~d} t_{2} .
$$

For $\theta=\eta=1$, we get

$$
\begin{aligned}
\mathbb{E}\left[X^{2}(t)\right]= & \frac{1}{2 a} e^{-a(2 t+1)} \\
& \times\left[\mathrm{E}_{1}(-2 a(t+1))-\mathrm{E}_{1}(-2 a)-2 \mathrm{e}^{2 a(t+1)}(\operatorname{Ei}(-a)-\operatorname{Ei}(-a(t+1)))\right. \\
& -2 \operatorname{Ei}(a(t+1))+\operatorname{Ei}(2 a(t+1))+2 \operatorname{Ei}(a)-\operatorname{Ei}(2 a)],
\end{aligned}
$$

where

$$
\operatorname{Ei}(z):=-\int_{-z}^{\infty} e^{-t} / t \mathrm{~d} t, \quad \mathrm{E}_{n}(z):=\int_{1}^{\infty} e^{-z t} / t^{n} \mathrm{~d} t .
$$

For $\theta=\eta=a=1$, we obtain

$$
\mathbb{E}\left[X^{2}(t)\right]=\mathrm{e}(-\operatorname{Ei}(-1)+\operatorname{Ei}(-1-t))+\mathrm{e}^{-(1+2 t)}(\operatorname{Ei}(1)-\operatorname{Ei}(1+t)) .
$$

(v) Dagum. If $C_{F}(\cdot)=C_{\mathscr{D}}(\cdot ; \delta, \epsilon)$, we cannot get an explicit formula for $\mathbb{E}\left[X^{2}(t)\right]$, but by numerical computation of (12) using Matlab we obtain the plots in Figure 2.

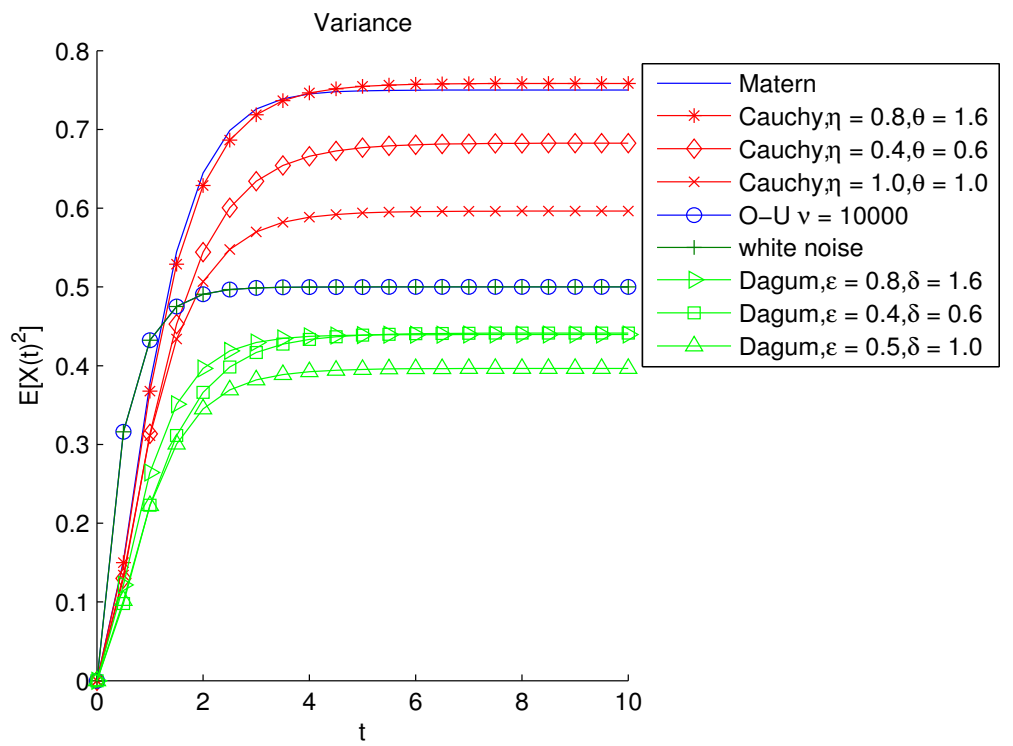

Figure 2. Variances under various forcings: Matérn, Cauchy $(\eta=0.8$, $\theta=1.6 ; \eta=0.4, \theta=0.6$; and $\eta=1.0, \theta=1.0)$, Ornstein-Uhlenbeck $(\nu=10,000)$, white noise, and Dagum $(\epsilon=0.8, \delta=1.6 ; \epsilon=0.4, \delta=0.6$; and $\epsilon=0.5, \delta=1.0)$. 


\section{Correlation structure of the response $X(\cdot)$}

The correlation function of the response can be readily calculated as follows (recall that, by construction, $\mathbb{E}[X(\cdot)]=0)$ :

$$
\begin{aligned}
C_{X}\left(t_{1}, t_{2}\right): & =\mathbb{E}\left[X\left(t_{1}\right) X\left(t_{2}\right)\right] \\
& =\mathbb{E}\left[\int_{0}^{t_{1}} Y\left(\tau_{1}\right) h_{a}\left(t_{1}-\tau_{1}\right) \mathrm{d} \tau_{1} \int_{0}^{t_{2}} Y\left(\tau_{2}\right) h_{a}\left(t_{2}-\tau_{2}\right) \mathrm{d} \tau_{2}\right] \\
& =\int_{0}^{t_{2}} \int_{0}^{t_{1}} \mathbb{E}\left[Y\left(\tau_{1}\right) Y\left(\tau_{2}\right)\right] h_{a}\left(t_{1}-\tau_{1}\right) h_{a}\left(t_{2}-\tau_{2}\right) \mathrm{d} \tau_{1} \mathrm{~d} \tau_{2} \\
& =\int_{0}^{t_{2}} \int_{0}^{t_{1}} \Psi\left(\tau_{1}, \tau_{2}\right) C_{F}\left(\tau_{1}, \tau_{2}\right) h_{a}\left(t_{1}-\tau_{1}\right) h_{a}\left(t_{2}-\tau_{2}\right) \mathrm{d} \tau_{1} \mathrm{~d} \tau_{2},
\end{aligned}
$$

where $C_{F}\left(\tau_{1}, \tau_{2}\right)$ is the covariance function of $F(t)$ and where

$$
\Psi\left(t_{1}, t_{2}\right):=\left(\beta+\gamma t_{1}\right)\left(\beta+\gamma t_{2}\right) U\left(t_{1}\right) U\left(t_{2}\right)
$$

(i) White noise. If $C_{F}=C_{\mathrm{WN}}$, the calculation of $C_{X}$ can be deduced from (13) and the fact that

$$
C_{Y}\left(t_{1}, t_{2}\right)=\Psi\left(t_{1}, t_{2}\right) C_{\mathrm{WN}}\left(t_{1}, t_{2}\right) .
$$

Also, keeping in mind that

$$
\delta\left(\tau_{1}-\tau_{2}\right)=0 \quad \text { if } \quad \tau_{1} \neq \tau_{2}
$$

if $t_{1}>t_{2}$, we see that

$$
\begin{aligned}
C_{X}\left(t_{1}, t_{2}\right)= & \int_{0}^{t_{2}} \int_{0}^{t_{1}} C_{Y}\left(\tau_{1}, \tau_{2}\right) h_{a}\left(t_{1}-\tau_{1}\right) h_{a}\left(t_{2}-\tau_{2}\right) \mathrm{d} \tau_{1} \mathrm{~d} \tau_{2} \\
= & \mathrm{e}^{-a\left(t_{1}+t_{2}\right)} \int_{0}^{t_{2}}\left(\beta+\gamma \tau_{2}\right)^{2} \mathrm{e}^{2 a \tau_{2}} \mathrm{~d} \tau_{2} \\
=\mathrm{e}^{-a\left(t_{1}+t_{2}\right)}\left\{\frac{\beta^{2}}{2 a}\left(\mathrm{e}^{2 a t_{2}}-1\right)+2 \beta \gamma\right. & {\left[\frac{\mathrm{e}^{2 a t_{2}}}{4 a^{2}}\left(2 a t_{2}-1\right)+\frac{1}{4 a^{2}}\right] } \\
& \left.+\frac{\gamma^{2}}{4 a^{3}}\left(\mathrm{e}^{2 a t_{2}}\left(2 a^{2} t_{2}^{2}-2 a t_{2}+1\right)-1\right)\right\} \\
= & \mathrm{e}^{-a\left(t_{1}-t_{2}\right)} \mathbb{E}\left[X\left(t_{2}\right)^{2}\right] .
\end{aligned}
$$

We can then repeat the same procedure when $t_{1}<t_{2}$ in order to deduce

$$
C_{X}\left(t_{1}, t_{2}\right)= \begin{cases}\mathrm{e}^{-a\left(t_{1}-t_{2}\right)} \mathbb{E}\left[X\left(t_{2}\right)^{2}\right] & \text { if } t_{1} \geqslant t_{2}, \\ \mathrm{e}^{-a\left(t_{2}-t_{1}\right)} \mathbb{E}\left[X\left(t_{1}\right)^{2}\right] & \text { if } t_{1}<t_{2} .\end{cases}
$$


From the equations above, if we let $\gamma=0$, we can see that when $t_{1}$ and $t_{2}$ are large enough,

$$
C_{X}\left(t_{1}, t_{2}\right) \approx C_{X}\left(t_{2}, t_{1}\right) \approx \mathrm{e}^{-a\left|t_{1}-t_{2}\right|} \frac{\beta^{2}}{2 a},
$$

which shows that the random process is homogeneous (that is, WSS). We see that this correlation function is different from the correlation function of white noise.

(ii) Ornstein-Uhlenbeck. In the case $F=O U$, we determine the correlation function of $X(t)$ as follows.

If $a=v=1$,

$$
C_{X}\left(t_{1}, t_{2}\right)= \begin{cases}\frac{1}{4}\left[\mathrm{e}^{-t_{1}+t_{2}}\left(t_{1}-t_{2}+1\right)+\mathrm{e}^{-t_{1}-t_{2}}\left(-t_{1}-t_{2}-1\right)\right] & \text { if } t_{1} \geqslant t_{2}, \\ \frac{1}{4}\left[\mathrm{e}^{-t_{2}+t_{1}}\left(t_{2}-t_{1}+1\right)+\mathrm{e}^{-t_{2}-t_{1}}\left(-t_{2}-t_{1}-1\right)\right] & \text { if } t_{1}<t_{2},\end{cases}
$$

which shows that $C_{X}$ is symmetric.

If $a=1, v \neq 1$, we get

$$
C_{X}\left(t_{1}, t_{2}\right)= \begin{cases}\frac{v \mathrm{e}^{-(1+v)\left(t_{1}+t_{2}\right)}}{2\left(-1+v^{2}\right)}\left[\mathrm{e}^{v t_{1}+t_{2}}+\mathrm{e}^{t_{1}+v t_{2}}-\mathrm{e}^{t_{1}+t_{2}+2 v t_{2}}\right. & \\ \left.+\mathrm{e}^{v\left(t_{1}+t_{2}\right)}\left(-1-v+v \mathrm{e}^{2 t_{2}}\right)\right] & \text { if } t_{1} \geqslant t_{2}, \\ C_{X}\left(t_{2}, t_{1}\right) & \text { if } t_{1}<t_{2} .\end{cases}
$$

Finally, if $a \neq 1$ and $a \neq v$,

$$
\begin{aligned}
C_{X}\left(t_{1}, t_{2}\right)=\frac{v}{2 a\left(a^{2}-v^{2}\right)}\left[a\left(\mathrm{e}^{-a\left(t_{1}+t_{2}\right)}-\mathrm{e}^{-v t_{1}-a t_{2}}-\mathrm{e}^{-a t_{1}-v t_{2}}+\mathrm{e}^{-v\left(t_{1}-t_{2}\right)}\right)\right. & \\
& +v\left(\mathrm{e}^{-a\left(t_{1}+t_{2}\right)}-\mathrm{e}^{-a\left(t_{1}-t_{2}\right)}\right] \quad \text { if } t_{1} \geqslant t_{2},
\end{aligned}
$$

and the symmetric extension follows when $t_{1}<t_{2}$.

Figure 3 shows the correlation function of the response from the OU process. Note that, as $v$ becomes large, the correlation function of $X(t)$ approaches the covariance of the response to the white noise excitation.

(iii) Matérn. If $C_{F}=C_{\mathcal{M}}(\cdot ; v=3 / 2)$, we can find the autocorrelation function of $X(t)$ by direct inspection. The correlation is symmetric, so we do not give the symmetry extensions for all the cases.

If $a \neq 1$, we easily get

$$
\begin{aligned}
C_{X}\left(t_{1}, t_{2}\right)= & \frac{1}{2 a\left(-1+a^{2}\right)^{2}}\left\{(-2+a)(1+a)^{2}\left[\mathrm{e}^{-2 a t_{2}}+\mathrm{e}^{-a\left(t_{1}+t_{2}\right)}-1\right]\right. \\
& +(-1+a)^{2}(2+a) \mathrm{e}^{-a\left(t_{1}-t_{2}\right)}-2 a\left(-3-t_{1}+a^{2}\left(1+t_{1}\right)\right) \mathrm{e}^{(-1+a) t_{1}-2 a t_{2}} \\
& -2 a\left[-3-t_{2}+a^{2}\left(1+t_{2}\right)\right] \mathrm{e}^{-\left(a t_{1}+t_{2}\right)} \\
& \left.-2 a\left[3+t_{1}-t_{2}+a^{2}\left(-1-t_{1}+t_{2}\right)\right] \mathrm{e}^{(-1+a)\left(t_{1}-t_{2}\right)}\right\} \quad \text { if } t_{1} \geqslant t_{2} .
\end{aligned}
$$




$$
\begin{aligned}
& \text { If } a=1, \\
& \qquad \begin{aligned}
C_{X}\left(t_{1}, t_{2}\right)= & \frac{1}{4}\left\{\mathrm{e}^{-t_{1}-t_{2}}\left[-3-3\left(t_{1}+t_{2}\right)-\left(t_{1}^{2}+t_{2}^{2}\right)\right]\right\} \\
& +\frac{1}{4} \mathrm{e}^{-\left(t_{1}-t_{2}\right)}\left[3+3\left(t_{1}-t_{2}\right)+\left(t_{1}-t_{2}\right)^{2}\right] \quad \text { if } t_{1} \geqslant t_{2} .
\end{aligned}
\end{aligned}
$$

If $t_{1}$ and $t_{2}$ are large enough,

$$
C_{X}\left(t_{1}, t_{2}\right) \approx \frac{1}{4} \mathrm{e}^{-\left(t_{1}-t_{2}\right)}\left[3+3\left(t_{1}-t_{2}\right)+\left(t_{1}-t_{2}\right)^{2}\right] \quad \text { if } t_{1} \geqslant t_{2},
$$
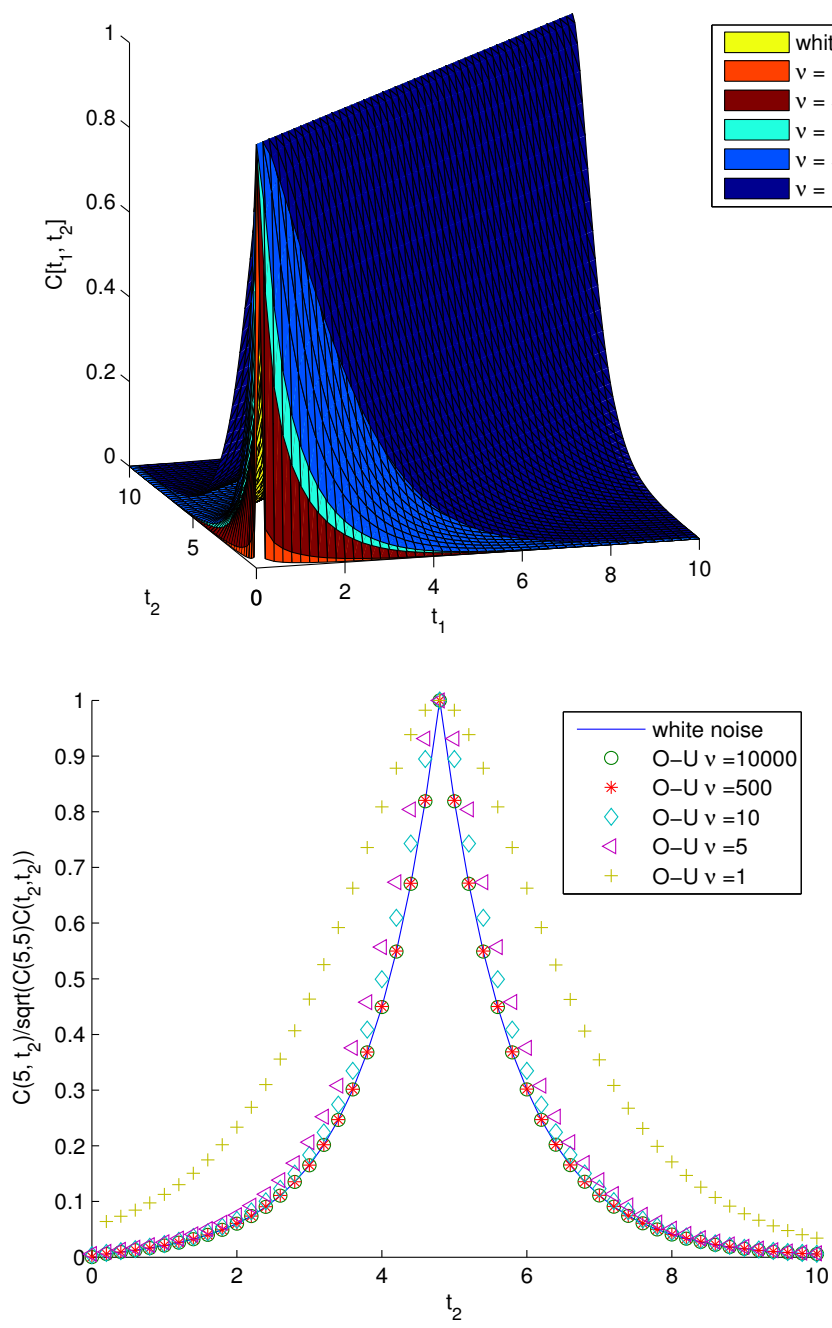

Figure 3. The correlation function under the OU forcings at various $v$ values (top) and the correlation functions of response $X(t)$ at $t_{1}=5$ under white noise and OU forcings (bottom). The white noise curve overlaps with the OU process curves for $v=10,000$ and 500 . 
and the random process is homogeneous, that is, $C_{X}\left(t_{1}, t_{2}\right)=C_{X}\left(\left|t_{2}-t_{1}\right|\right)$. We also observe that, if the excitation correlation function is Matérn, the correlation function of the response is approximately Matérn.

(iv) Generalized Cauchy. If $C_{F}(\cdot)=C_{\mathscr{C}}(\cdot, \theta, \eta)$ then, if the correlation function of $F(t)$ is Cauchy, we can find the correlation function of $X(t)$ by direct inspection:

$$
\begin{aligned}
C_{X}\left(t_{1}, t_{2}\right)= & \frac{1}{2} \mathrm{e}^{-1-t_{1}-t_{2}}\left[2 \operatorname{Ei}(1)-\operatorname{Ei}\left(1+t_{2}\right)-\operatorname{Ei}\left(1+t_{1}\right)\right] \\
& +\frac{1}{2} \mathrm{e}^{1-\left(t_{1}-t_{2}\right)}\left[-\operatorname{Ei}(-1)+\operatorname{Ei}\left(-1-t_{2}\right)\right] \\
& +\frac{1}{2} \mathrm{e}^{-1-\left(t_{1}-t_{2}\right)}\left[-\operatorname{Ei}(1)+\operatorname{Ei}\left(1+t_{1}-t_{2}\right)\right] \\
& +\frac{1}{2} \mathrm{e}^{1+\left(t_{1}-t_{2}\right)}\left[\operatorname{Ei}\left(-1-t_{1}\right)-\operatorname{Ei}\left(-1-t_{1}+t_{2}\right)\right] \text { if } t_{1} \geqslant t_{2} .
\end{aligned}
$$

Once again, we omit the case $t_{1}<t_{2}$ since it can be deduced by a symmetry extension. Note that, although $\operatorname{Ei}\left(1+t_{1}\right)$ and $\operatorname{Ei}\left(1+t_{2}\right)$ go to $+\infty$ when $t_{1}, t_{2} \rightarrow$ $+\infty$, the function $\mathrm{e}^{-1-t_{1}-t_{2}}$ decreases more rapidly. Hence, when $t_{1}$ and $t_{2}$ are large enough, the first term goes to zero. When $t_{1}, t_{2} \rightarrow+\infty$, the functions $\operatorname{Ei}\left(-1-t_{1}\right)$ and $\operatorname{Ei}\left(-1-t_{2}\right)$ are close to zero as well. Therefore, we have

$$
\begin{aligned}
C_{X}\left(t_{1}, t_{2}\right) \approx \frac{1}{2} \mathrm{e}^{1-\left(t_{1}-t_{2}\right)}[-\operatorname{Ei}(-1)]+ & \frac{1}{2} \mathrm{e}^{-1-\left(t_{1}-t_{2}\right)}\left[-\operatorname{Ei}(1)+\operatorname{Ei}\left(1+t_{1}-t_{2}\right)\right] \\
& +\frac{1}{2} \mathrm{e}^{1+\left(t_{1}-t_{2}\right)}\left[-\operatorname{Ei}\left(-1-t_{1}+t_{2}\right)\right] \quad \text { if } t_{1} \geqslant t_{2} .
\end{aligned}
$$

Now we see that

$$
\begin{aligned}
& C_{X}\left(t_{1}, t_{2}\right)= C_{X}\left(t_{2}, t_{1}\right) \approx C_{X}\left(\left|t_{1}-t_{2}\right|\right) \\
&=\frac{1}{2} \mathrm{e}^{1-\left|t_{1}-t_{2}\right|}[-\operatorname{Ei}(-1)]+\frac{1}{2} \mathrm{e}^{-1-\left|t_{1}-t_{2}\right|}\left[-\operatorname{Ei}(1)+\operatorname{Ei}\left(1+\left|t_{1}-t_{2}\right|\right)\right] \\
&+\frac{1}{2} \mathrm{e}^{1+\left|t_{1}-t_{2}\right|}\left[-\operatorname{Ei}\left(-1-\left|t_{1}-t_{2}\right|\right)\right],
\end{aligned}
$$

or

$$
\begin{aligned}
C_{X}\left(t_{1}, t_{2}\right)= & C_{X}\left(t_{2}, t_{1}\right) \approx C_{X}(r) \\
= & \frac{1}{2} \mathrm{e}^{1-r}[-\operatorname{Ei}(-1)]+\frac{1}{2} \mathrm{e}^{-1-r}[-\operatorname{Ei}(1)+ \\
& =\frac{\operatorname{Ei}(1+r)]}{2} \mathrm{e}^{1+r}[-\operatorname{Ei}(-1-r)],
\end{aligned}
$$

which means that, when $t_{1}$ and $t_{2}$ are large enough, the response is homogeneous. Finally, if $r \rightarrow 0$, from Taylor's formula we have

$$
\begin{aligned}
& C_{X}(r) \\
& =-\mathrm{eEi}(-1)+\left(-\frac{1}{2}-\frac{1}{2} \mathrm{eEi}(-1)\right) r^{2}+\frac{1}{6} r^{3}+\left(-\frac{1}{8}-\frac{1}{24} \mathrm{eEi}(-1)\right) r^{4}+\frac{7}{120} r^{5}+O\left(r^{6}\right) .
\end{aligned}
$$

Comparing with the Cauchy function,

$$
\frac{1}{1+r}=1-r+r^{2}-r^{3}+r^{4}-r^{5}+O\left(r^{6}\right),
$$

we see that the response from Cauchy excitation is not Cauchy. 


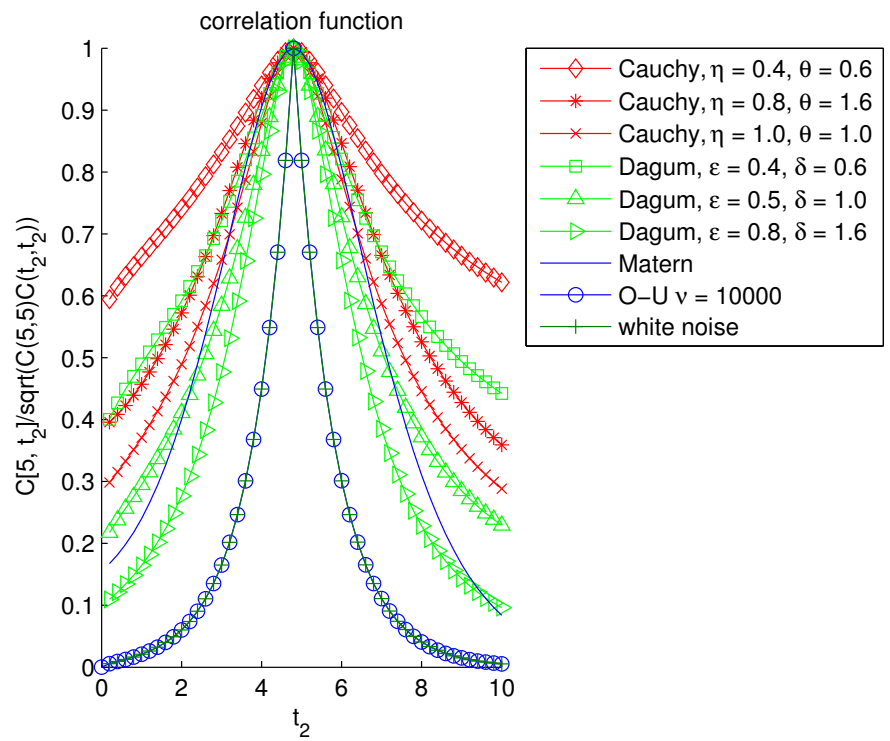

Figure 4. The correlation functions of response $X(t)$ at $t_{1}=5$ under various forcings: Cauchy $(\eta=0.4, \theta=0.6 ; \eta=0.8, \theta=1.6$; and $\eta=1.0, \theta=1.0)$, Dagum $(\epsilon=0.4, \delta=0.6 ; \epsilon=0.5, \delta=1.0$; and $\epsilon=0.8, \delta=1.6)$, Matérn, Ornstein-Uhlenbeck $(v=10,000)$, and white noise.

(v) Dagum. In this case no explicit analytical formula for the correlation function of $X(t)$ can be obtained and one has to proceed by numerical integration of (13). Figure 4 shows the resulting correlation function compared to those due to the four other excitations. Since there is no formula analogous to (14), we cannot say whether the response from Dagum-type excitation is Dagum or not.

\section{Conclusions}

A study has been conducted of the responses of first-order, linear dynamical systems under time-stationary random forcings of Cauchy and Dagum types. These forcings lack explicit parametric spectral densities, yet they allow the decoupling of the fractal dimension and Hurst effect. Working directly in the time domain, we find transient second-order characteristics of responses and, for comparison, we also examine the effects of Gaussian white noise, Ornstein-Uhlenbeck (which in the limit becomes white noise), and Matérn forcings. Overall, given the same variance on input, the variance on output is strongest for Matérn, then Cauchy, then white noise, and finally Dagum forcing. We also find that, if the excitation correlation function is Matérn, the correlation function of the response is approximately Matérn. On the other hand, the response due to the Cauchy excitation is not Cauchy, but, at this stage, we cannot say whether the response due to the Dagum 
excitation (with its fractal and Hurst effects) is Dagum or not. The latter issue will require further research. An analogous study of the responses of second-order, linear dynamical systems subjected to Cauchy and Dagum excitations is presently underway [Shen et al. 2014b].

While the studies reported in the aforementioned paper and in the present work focused on randomness in the time domain for a one-degree-of-freedom system, similar studies have been conducted in the spatial domain for static systems. Namely, responses of elastic rods (or, equivalently, shear beams) [Shen et al. 2015] and Bernoulli-Euler beams [Shen et al. 2014a] with random field properties and, also possibly, under random field forcings of either Cauchy or Dagum type have been compared with those of either linear, exponential, or Matérn. Typically, given the same variance of the random field, the variance on output is strongest for Matérn. However, the relative effects of Dagum, Cauchy, linear, and exponential models depend on the particular loading situation. In a number of cases, the results may be obtained in explicit (albeit very lengthy) analytical forms, but as Cauchy and Dagum models are introduced, one has to resort to numerics. Thus, while the introduction of fractal and Hurst effects brings more reality into models of randomness in time and space domains, it results in more challenging analyses.

Further research is needed in order to evaluate the impact of the proposed framework in terms of the fractal dimension and Hurst effect for the resulting stochastic structures. Analytically, this is not an easy task. From a statistical viewpoint, it would be of interest to follow along the lines of [Gneiting and Schlather 2004; Mateu et al. 2007]: first, simulating Gaussian random processes under the covariances obtained in the present paper, then estimating the fractal dimension and Hurst effect, and inspecting whether there is any tendency toward decoupling. This will be an important issue to address in the future.

\section{Acknowledgements}

Lihua Shen was partially supported by the National Science Foundation of China under Grant 11171232 and the Beijing Municipal Education Commission under Grant KZ201310028030. Martin Ostoja-Starzewski was partially supported by the NSF under grant CMMI-1030940, and Emilio Porcu by Proyecto Fondecyt Regular number 1130647.

\section{References}

[Adler 1981] R. J. Adler, The geometry of random fields, Wiley, Chichester, 1981. Reprinted in Classics in Applied Mathematics 62, SIAM, Philadelphia, 2010.

[CFA 2008] CFA, Context free art: tutorials/fractals, 2008, http://www.contextfreeart.org/mediawiki/ index.php/Tutorials/Fractals. 
[Christakos 2000] G. Christakos, Modern spatiotemporal geostatistics, Studies in Mathematical Geology 6, Oxford University Press, New York, 2000.

[Elishakoff 1983] I. Elishakoff, Probabilistic methods in the theory of structures, Wiley, New York, 1983. 2nd ed. published by Dover, Mineola, NY, 1999.

[Gneiting and Schlather 2004] T. Gneiting and M. Schlather, "Stochastic models that separate fractal dimension and the Hurst effect", SIAM Rev. 46:2 (2004), 269-282.

[Hall and Wood 1993] P. Hall and A. Wood, "On the performance of box-counting estimators of fractal dimension”, Biometrika 80:1 (1993), 246-252.

[Matérn 1986] B. Matérn, Spatial variation, 2nd ed., Lecture Notes in Statistics 36, Springer, Berlin, 1986.

[Mateu et al. 2007] J. Mateu, E. Porcu, and O. Nicolis, "A note on decoupling of local and global behaviours for the Dagum random field", Probab. Eng. Mech. 22:4 (2007), 320-329.

[Matheron 1965] G. Matheron, Les variables régionalisées et leur estimation, Masson, Paris, 1965.

[Porcu and Stein 2012] E. Porcu and M. L. Stein, "On some local, global and regularity behaviour of some classes of covariance functions", pp. 221-238 in Advances and challenges in space-time modelling of natural events, edited by E. Porcu et al., Lecture Notes in Statistics 207, Springer, Heidelberg, 2012.

[Porcu et al. 2007] E. Porcu, J. Mateu, A. Zini, and R. Pini, "Modelling spatio-temporal data: a new variogram and covariance structure proposal”, Stat. Probab. Lett. 77:1 (2007), 83-89.

[Ruiz-Medina et al. 2011] M. D. Ruiz-Medina, E. Porcu, and R. Fernandez-Pascual, "The Dagum and auxiliary covariance families: towards reconciling two-parameter models that separate fractal dimension and the Hurst effect", Probab. Eng. Mech. 26:2 (2011), 259-268.

[Shen et al. 2014a] L. Shen, M. Ostoja-Starzewski, and E. Porcu, "Bernoulli-Euler beams with random field properties under random field forcings: fractal and Hurst effects", Arch. Appl. Mech. 84 (2014), 1595-1626.

[Shen et al. 2014b] L. Shen, M. Ostoja-Starzewski, and E. Porcu, "Harmonic oscillator driven by random processes with fractal and Hurst effects", submitted, 2014.

[Shen et al. 2015] L. Shen, M. Ostoja-Starzewski, and E. Porcu, "Elastic rods and shear beams with random field properties under random field loads: fractal and Hurst effects", J. Engin. Mech. (ASCE) (2015).

[Stein 1999] M. L. Stein, Interpolation of spatial data: some theory for Kriging, Springer, New York, 1999.

Received 15 Apr 2013. Revised 14 Sep 2013. Accepted 17 Nov 2013.

LIHUA SHEN: shenlh@lsec.cc.ac.cn

Department of Computational Mathematics, Capital Normal University, Beijing, 100037, China

MARTIN OsTOJA-STARZEWSKI: martinos@illinois.edu

Department of Mechanical Science and Engineering, University of Illinois at Urbana-Champaign, 1206 W. Green Street, Urbana, IL 61801-2906, United States

EMILIO PORCU: emilio.porcu@usm.cl

Department of Mathematics, University Federico Santa Maria, 2360102 Valparaíso, Chile 


\section{EDITORIAL BOARD}

ANTONIO CARCATERRA ERIC A. CARLEN

FRANCESCO DELL'ISOLA

RAFFAELE ESPOSITO

ALBERT FANNJIANG

Gilles A. FrancFort

PiERANGElo MARCATI

JEAN-JACQUES MARIGO

PETER A. MARKOWICH MARTIN OSTOJA-STARZEWSKI

PIERRE SEPPECHER

DAVID J. STEIGMANN

PAUl STEINMANN

PierRe M. Suquet

\section{MANAGING EDITORS}

MICOL AMAR

CORRADO LATTANZIO

ANGELA MADEO

MARTIN OSTOJA-STARZEWSKI

\section{ADVISORY BOARD}

ADNAN AKAY

Holm AltenbaCH

MICOL AMAR

HARM ASKES

TEODOR ATANACKOVIĆ

VICTOR BERDICHEVSKY

GuY BouchitTÉ

ANDREA BRAIDES

ROBERTO CAMASSA

MAURO CARFORE

ERIC DARVE

FELIX DARVE

ANNA DE MASI

Gianpietro Del Piero

EMMANUELE Di BENEDETTO

BERNOLD FIEDLER

IRENE M. GAMBA

SERGEY GAVRILYUK

TIMOTHY J. HEALEY

DOMINIQUE JEULIN

ROGER E. KHAYAT

CORRADO LATTANZIO

ROBERT P. LIPTON

ANGELO LUONGO

ANGEla MadeO

JUAN J. MANFREDI

CARLO MARCHIORO

GÉrard A. MAUGin

ROBERTO NATALINI

PATRIZIO NEFF

ANDREY PIATNITSKI

ERrico Presutti

MARIO PUlVIRENTI

LuCiO RuSSO

Miguel A. F. SANJUAN

Patrick SElvadurai

ALEXANDER P. SEYRANIAN

MIROSLAV ŠILHAVÝ

GUIDO SWEERS

ANTOINETTE TORDESILLAS

LEV TRUSKINOVSKY

JUAN J. L. VELÁZQUEZ

VINCENZO VESPRI

ANGELO VULPIANI msp.org/memocs

Università di Roma “La Sapienza”, Italia

Rutgers University, USA

(CO-CHAIR) Università di Roma "La Sapienza", Italia

(TREASURER) Università dell'Aquila, Italia

University of California at Davis, USA

(CO-CHAIR) Université Paris-Nord, France

Università dell' Aquila, Italy

École Polytechnique, France

DAMTP Cambridge, UK, and University of Vienna, Austria

(CHAIR MANAGING EDITOR) Univ. of Illinois at Urbana-Champaign, USA

Université du Sud Toulon-Var, France

University of California at Berkeley, USA

Universität Erlangen-Nürnberg, Germany

LMA CNRS Marseille, France

Università di Roma “La Sapienza”, Italia

Università dell' Aquila, Italy

Université de Lyon-INSA (Institut National des Sciences Appliquées), France (CHAIR MANAGING EDITOR) Univ. of Illinois at Urbana-Champaign, USA

Carnegie Mellon University, USA, and Bilkent University, Turkey

Otto-von-Guericke-Universität Magdeburg, Germany

Università di Roma "La Sapienza”, Italia

University of Sheffield, UK

University of Novi Sad, Serbia

Wayne State University, USA

Université du Sud Toulon-Var, France

Università di Roma Tor Vergata, Italia

University of North Carolina at Chapel Hill, USA

Università di Pavia, Italia

Stanford University, USA

Institut Polytechnique de Grenoble, France

Università dell'Aquila, Italia

Università di Ferrara and International Research Center MEMOCS, Italia

Vanderbilt University, USA

Freie Universität Berlin, Germany

University of Texas at Austin, USA

Université Aix-Marseille, France

Cornell University, USA

École des Mines, France

University of Western Ontario, Canada

Università dell' Aquila, Italy

Louisiana State University, USA

Università dell' Aquila, Italia

Université de Lyon-INSA (Institut National des Sciences Appliquées), France

University of Pittsburgh, USA

Università di Roma "La Sapienza", Italia

Université Paris VI, France

Istituto per le Applicazioni del Calcolo "M. Picone", Italy

Universität Duisburg-Essen, Germany

Narvik University College, Norway, Russia

Università di Roma Tor Vergata, Italy

Università di Roma "La Sapienza", Italia

Università di Roma “Tor Vergata", Italia

Universidad Rey Juan Carlos, Madrid, Spain

McGill University, Canada

Moscow State Lomonosov University, Russia

Academy of Sciences of the Czech Republic

Universität zu Köln, Germany

University of Melbourne, Australia

École Polytechnique, France

Bonn University, Germany

Università di Firenze, Italia

Università di Roma La Sapienza, Italia

MEMOCS (ISSN 2325-3444 electronic, 2326-7186 printed) is a journal of the International Research Center for the Mathematics and Mechanics of Complex Systems at the Università dell'Aquila, Italy.

Cover image: "Tangle" by @ John Horigan; produced using the Context Free program (contextfreeart.org).

PUBLISHED BY

mathematical sciences publishers

nonprofit scientific publishing

http://msp.org/

(C) 2015 Mathematical Sciences Publishers 
Mathematics and Mechanics of Complex Systems vol. 3 no. 1

Effects of damping on the stability of the compressed

Nicolai beam

Angelo Luongo, Manuel Ferretti and Alexander P. Seyranian

Responses of first-order dynamical systems to Matérn, Cauchy, and Dagum excitations

Lihua Shen, Martin Ostoja-Starzewski and Emilio Porcu

Reflections on mathematical models of deformation waves in elastic microstructured solids

Jüri Engelbrecht and Arkadi Berezovski

On the approximation theorem for structured deformations from $B V(\Omega)$

Miroslav Šilhavý

MEMOCS is a journal of the International Research Center for the Mathematics and Mechanics of Complex Systems at the Università dell' Aquila, Italy.

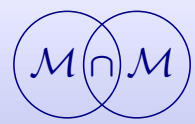

